\title{
Musarañas del género Cryptotis (Eulipotyphla: Soricidae) en el Pleistoceno Tardío de los Andes Ecuatorianos
}

\author{
Pablo A. Moreno Cárdenas, José Luis Román-Carrión
}

Pablo A. Moreno Cárdenas pablo.moreno@biodiversidad.gob.ec

Mastozoología del Instituto Nacional de Biodibersidad (MECN). Pasaje Rumipamba 341 y Av. de los Shyris. Código Postal 170515. Quito - Ecuador.

Mastozoología del Instituto de Ciencias Biológicas de la Escuela Politécnica Nacional (MEPN). Avenida Ladrón de Guevara E-1 1 253 e Isabel la Católica. Apartado 17-012759. Quito - Ecuador.

\section{José Luis Román-Carrión}

Sección de Paleontología del Instituto de Ciencias Biológicas de la Escuela Politécnica Nacional (MEPN). Avenida Ladrón de Guevara E-11 253 e Isabel la Católica. Apartado 17-01-2759. Quito - Ecuador.

BOL. SOC. GEOL. MEX. 2017

VOL. 69 NO. 2

P. $421-432$

\section{RESUMEN}

Durante una excavación paleontológica en la Quebrada Chalán, provincia de Chimborazo, se registraron cinco fémures, cuatro húmeros, ocho huesos pélvicos, dos incisivos superiores y un fragmento de maxilar de Cryptotis, con las siguientes piezas dentarias: $\mathrm{P} 4, \mathrm{M} 1$ y M2. Los fósiles fueron colectados en los niveles superiores de la Formación Cangagua asignada al pleistoceno superior. En la fase de análisis se tomaron nueve medidas de los húmeros y los fémures comparándose con los de las especies ecuatorianas actuales. Los fósiles fueron asignados a la especie Cryptotis niausa, principalmente por la forma arqueada del segmento anterior de la fosa mesopterigoidea, así como las dimensiones similares a las de $C$. niausa en fémures, húmeros y maxilar. Durante el pleistoceno tardío C. niausa se distribuyó 160 $\mathrm{km}$ al sur de su ubicación actual. Sabemos que hoy, esta especie no está en la localidad del hallazgo paleontológico, debido a que su clima es más caliente y seco respecto a los páramos de pajonal húmedo donde habita actualmente. Este hallazgo demuestra que la localidad de Quebrada Chalán, tenía otro tipo de vegetación a finales del pleistoceno tardío durante el segundo y tercer interglaciar, similar a los páramos actuales cuyo pajonal se encuentran a partir de los 3200 $\mathrm{m}$. El análisis morfológico del material fósil de C. niausa muestra una estructura ósea y muscular más robusta que los individuos de las poblaciones actuales. Algunos individuos fósiles presentan dimensiones más anchas de los fémures y los húmeros, asociadas a su mayor capacidad de cavar sus madrigueras y canales en suelos endurecidos producto de la marcada actividad volcánica.

Palabras clave: Ghimborazo, Guaternario, Post-cráneo, Quebrada Chalán, Valle Interandino.

\section{ABSTRACT}

During a paleontological excavation in Chalán Gorge (Chimborazo province), some fossil of micromammals where registered, including, five femurs, four humerus, eight pelvic bones and a fragment of maxilla of Cryptotis, with P4, M1 and M2 dental pieces. The fossils where collected in higher levels of cangagua formation of upper pleistocene. Nine measures of humerus and femurs fossil where obtained, and compared with the current Ecuadorian species. The results showed that the fossils belonged to Cryptotis niausa species, by the arcuate shape of the anterior segment of the mesopterygoid fossa, as well as similar dimensions in femurs, humerus and maxillary bone of $\underline{C}$. niausa. In the upper pleistocene $\underline{C}$. niausa was distributed $160 \mathrm{~km}$ south of its current distribution. This species is not currently distributed in the find locality, because the climate is warmer and drier than the wet Andean grasslands where C. niausa now lives. This finding demonstrates that Chalán Gorge had other vegetation in the late pleistocene during the second and third interglacial ages, similar to current Andean grasslands whose "pajonales" are above 3200 meters. Morphological analysis of fossil C. niausa showes individuals with bones structure and muscle more robust than the current populations. Some Crrpptotis fossils individuals have wider dimensions of the femurs and humerus, associated with an increased ability to dig their burrowes and channels on hard soils result of strong volcanic activity.

Keyzords: Chimborazo, Quaternary, Post-cranium, Chalan Gorge, Interandean Valley. 


\section{Introducción}

En América Central y América del Norte, se han realizado algunos estudios sobre fósiles de musarañas: Findley (1953) registra fósiles de Cryptotis en la caverna de San Josecito en México, que posteriormente serían identificadas como C. mexicanus y C. parous, más grandes que los individuos actuales (Esteva et al., 2005). Harris $(1989,1997)$ registró en 12 sectores de Nuevo México, datados con radiocarbono entre 22000 hasta 55000 años, algunas especies de musarañas del género Sorex (S. merriami, $S$. montanus, $S$. nanus y $S$. plebei) y de la musaraña menor (Cryptotis parva).

Otros estudios muestran que algunas especies actuales de Cryptotis decrecieron en tamaño, a partir del pleistoceno tardío, como es el caso de Cryptotis mexicanus (Esteva et al., 2005). Por otro lado existen registros fósiles del mismo género que experimentaron cambios en dirección contraria, aumentando de tamaño desde el pleistoceno tardío hasta la época actual, como es el caso de Cryptotis goodwini que en la actualidad es una especie de mayor talla a la de sus registros fósiles (Woodman y Croft, 2005).

Ya en Sudamérica, de Porta (1965) menciona restos subrecientes de Cryptotis, en el sedimento de relleno de una grieta en rocas calizas, mezclado inclusive con fauna marina del cretácico, en la localidad colombiana de Curití en el Departamento de Santander, sin contar con una datación estratigráfica y radiométrica fiable. En otros trabajos realizados por Fejfar et al. (1993, 1996) reportan de manera general registros holocénicos de Cryptotis en las localidades de Cuesaca y La Calera, al norte de Ecuador en la provincia del Carchi.

Debido a que la mayoría de las especies de micromamíferos que habitaron en el pleistoceno tardío habitan aún en la actualidad y ocupan hábitats específicos. Dichos micromamíferos son utilizados como instrumentos para reconstruir los paleoambientes (Andrews, 1990), caso contrario a la megafauna que se extinguió prácticamente en su totalidad (Harris, 1997; Román-Carrión, 2010,
Román-Carrión y Lara, 2011; Sánchez et al., 2013).

En este trabajo reportamos los primeros fósiles datados e identificados de Cryptotis de Sudamérica, colectados en la Formación Cangagua, definida por Sauer (1950) como un estrato de cenizas endurecidas, de origen eólico, fluvial o lacustre, asignadas al Pleistoceno Superior, edad mamífero Lujanense (SALMA), caracterizada además por la presencia de los icnofósiles: Coprinisphaera murguiai y C. quitu (Sánchez et al., 2013), asignada al pleistoceno superior.

\section{2. Área de estudio}

El sitio de estudio se encuentra al sur del cantón Riobamba en la provincia de Chimborazo (Figura 1). Sus quebradas son parte de la cuenca del río Chambo que fluye hacia la cuenca amazónica. En la actualidad el yacimiento de Quebrada Chalán se encuentra en un ecosistema de arbustales estacionales a 2800 metros de altitud (Josse et al., 2009), rodeado de campos de cultivos pertenecientes a diferentes comunidades indígenas.

Los fósiles de Cryptotis fueron colectados por Alejandro Mesías y José Luis Román entre los años 2009 y 2012, en el relleno de dormideros de aves rapaces, dentro de la cangagua eólica en la parte superior de Quebrada Chalán (01 46’32.3” S / $\left.78^{\circ} 37^{\prime} 41.1 " \mathrm{~W}\right)$.

\section{Métodos}

Se identificaron cinco fémures, cuatro húmeros, ocho huesos pélvicos, dos incisivos superiores y un fragmento de maxilar de Cryptotis, con P4, M1 y M2 izquierdos completos. Los húmeros y fémures fósiles fueron analizados y comparados con tres ejemplares de Cryptotis montivagus, siete ejemplares de Cryptotis niausa, dos de Cryptotis equatoris y uno de Cryptotis osgoodi de la colección de mastozoología 


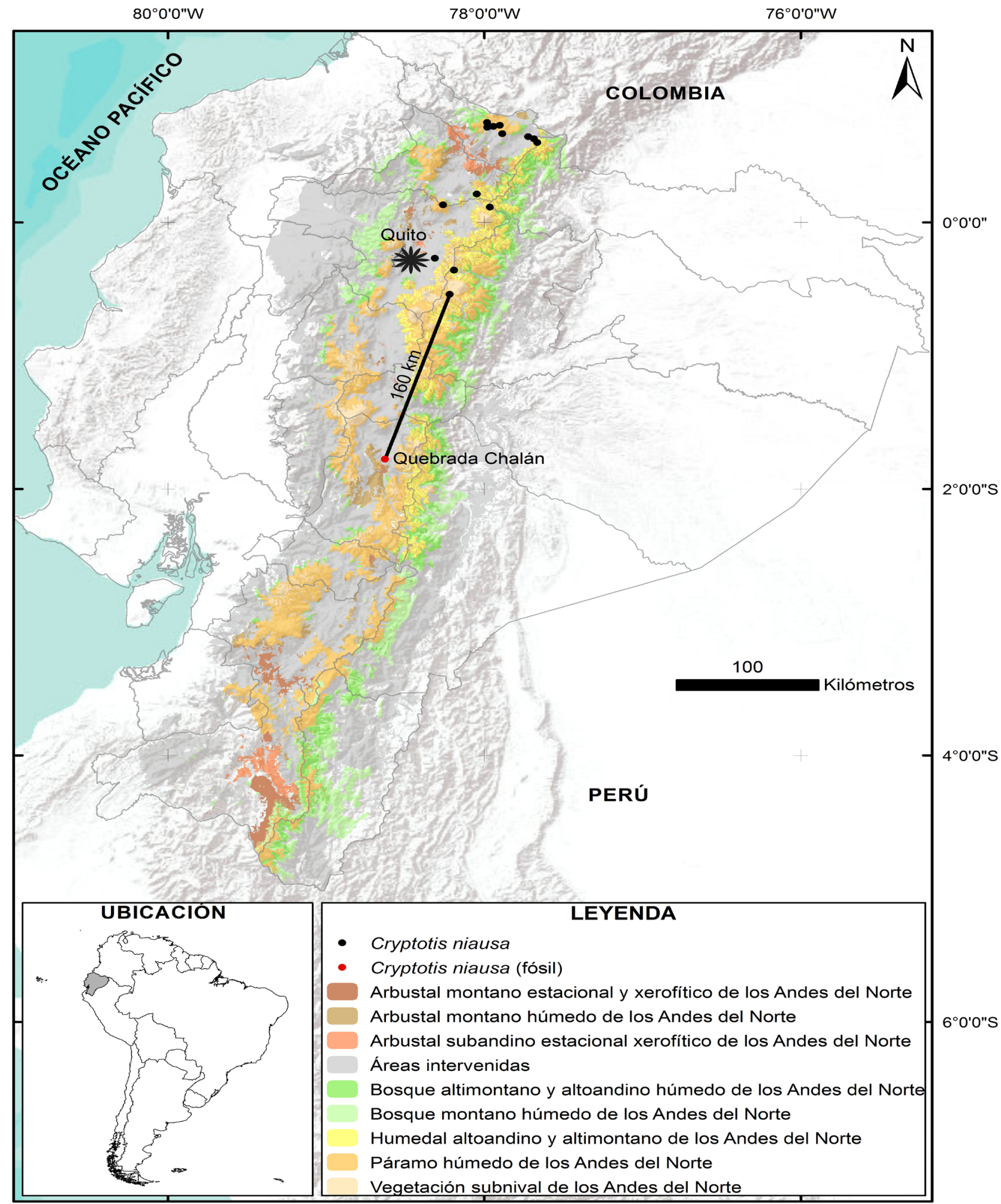

Figura 1 Ubicación del registro fósil de $C$. niausa (quebrada Chalán), junto a la distribución actual de esta especie en los ecosistemas de páramos andinos del norte y centro de Sudamérica (Josse et al., 2009). Se aprecia que en la actualidad Chalán se encuentra en un ecosistema de arbustales estacionales. 
del Instituto de Ciencias Biológicas de la Escuela Politécnica Nacional del Ecuador (MEPN).

Se obtuvieron nueve medidas de los húmeros y fémures de las cuatro especies actuales mencionadas y del fósil, siguiendo la metodología de Woodman y Gaffney (2014) y Woodman y Stabile (2015). Las medidas se describen a continuación: $\mathrm{LH}=$ Longitud del húmero; LCDP = Longitud de la cresta deltopectoral; $\mathrm{AEH}=$ Ancho epicondilar del húmero; $\mathrm{DMH}=$ Diámetro medio-lateral del húmero; $\mathrm{LF}=$ Longitud del fémur; $\mathrm{AEF}=$ Ancho epicondilar del fémur; DMF = Diámetro medio-lateral del fémur. Además se obtuvo, la distancia existente entre el tubérculo mayor y el menor del húmero (inter-tubercular); y la distancia entre el tercer trocánter y el trocánter menor del fémur (inter-trocanter). Se aplicó el índice epicóndilo-femoral (IEF), que es la relación entre el ancho epicondilar del fémur (AEF) dividida para la longitud del fémur (LF); y que indica el área relativa donde se originan los músculos flexores de los dedos del pie, los extensores del pie, además de los músculos retractores y aductores del fémur; siendo este índice más alto en los micromamíferos que tienen hábitos más excavadores y menos en los ambulatorios, según Woodman y Gaffney (2014). Además, la distribución espacial en puntos de las medidas de LH, AEH e inter-tubercular de las especies de Cryptotis de Ecuador para conocer la capacidad cavadora de cada especie.

Las medidas fueron obtenidas con un calibrador digital $0.1 \mathrm{~mm}$ de aproximación. Se aplicó un análisis de varianza de un factor (ANOVA), para conocer la significancia entre las medidas de los húmeros y fémures, usando el programa SPSS 18 (SPSS, 2009) y además un análisis de componentes principales (PCA) con el programa PAST3 (Hammer, 2001) mediante una matriz de correlaciones, para conocer cómo se distribuyen los caracteres de cada especie actual y fósil.

Análisis del material fósil de este yacimiento, datados mediante el análisis espectrométrico de masas con aceleradores (AMS) de ${ }^{13} \mathrm{C} /{ }^{12} \mathrm{C}$, dieron como resultado que las muestras tienen una antigüedad de 40630 ( \pm 410$)$ años antes del presente. El Ma- terial colectado se encuentra depositado en la colección de Paleontología del Instituto de Ciencias Biológicas de la Escuela Politécnica Nacional en Quito con las siglas EPNV.

\section{Resultados}

Los análisis estadísticos como morfológicos de los fósiles indicaron que los restos encontrados corresponden con la especie C. niausa (Moreno y Albuja, 2014), actualmente distribuida en los páramos andinos del norte de Ecuador (Figura 1); mientras que el registro fósil se encuentra a $160 \mathrm{~km}$ al sur de la distribución más austral de esta especie que hasta el momento es la localidad de Papallacta en la provincia del Napo.

En el PCA, los componentes 1 y 2 explicaron el $79 \%$ de la varianza. En el componente 1 se aprecian las dimensiones de ancho de los húmeros y fémures y en el componente 2 las principales medidas de longitud (Tabla 1).

Las dimensiones de los restos fósiles de Cryptotis del sector de Chalán se sobreponen con las de $C$. niausa actuales. Sin embargo se nota que los fémures de C. niausa fósil son mayores en longitud total, ancho epicondilar, ancho inter-trocanter y diámetro medio del fémur (Figura 2). También se aprecia que los componentes principales de C. montivagus, C. equatoris y $C$. osgoodi no se sobreponen en las distribuciones; siendo C. osgoodi la especie con las menores dimensiones en los húmeros y fémures, mientras que $C$. niausa tiene mayores dimensiones en la longitud del húmero.

Los resultados del ANOVA indican que la longitud de húmeros y fémures de las especies de Cryptotis de Ecuador mostraron únicamente diferencias significativas en las medidas de ancho (Tabla 2), especialmente en AEF, inter-trocánter y DMF. Las características de los húmeros y los fémures de las especies de Cryptotis ecuatorianas arrojaron algunas pistas claves sobre los hábitos de movilización y de la capacidad cavadora en sus hábitats, ya que mientras más cortos los húmeros y los fémures en las especies de micromamíferos cavadores 
Tabla 1. Análisis de componentes principales (PCA), con los tres componentes que explicaron el $86 \%$ de la varianza total para cada variable.

\begin{tabular}{|l|r|r|r|}
\hline \multicolumn{1}{|c|}{ Medidas } & CP 1 & CP 2 & CP 3 \\
\hline LH & 0.271 & 0.651 & -0.053 \\
\hline LCDP & 0.269 & 0.592 & -0.157 \\
\hline AEH & 0.339 & -0.126 & -0.367 \\
\hline DMH & 0.375 & -0.06 & -0.217 \\
\hline inter-tubercular & 0.339 & -0.079 & -0.022 \\
\hline LF & 0.277 & 0.103 & 0.882 \\
\hline AEF & 0.378 & -0.217 & -0.05 \\
\hline DMF & 0.35 & -0.309 & 0.093 \\
\hline inter-trocánter & 0.378 & -0.216 & 0.031 \\
\hline Varianza & 67.679 & 10.911 & 7.032 \\
\hline Autovalor & 6.091 & 0.982 & 0.633 \\
\hline
\end{tabular}

Tabla 2. ANOVA de un factor de las nueve medidas de los húmeros y fémures de Cryptotis de Ecuador.

\begin{tabular}{|l|c|c|c|c|}
\hline \multicolumn{1}{|c|}{ Medidas } & Suma de cuadrados & gl & F & Sig. \\
\hline LH & 0.565 & 17 & 1.419 & 0.300 \\
\hline LCDP & 0.461 & 17 & 2.048 & 0.178 \\
\hline AEH & 1.057 & 17 & 20.428 & $<0.001$ \\
\hline DMH & 0.094 & 17 & 19.746 & $<0.001$ \\
\hline inter-tubercular & 0.217 & 17 & 13.864 & 0.001 \\
\hline LF & 0.602 & 17 & 2.068 & 0.175 \\
\hline AEF & 0.607 & 17 & 211.810 & $<0.001$ \\
\hline DMF & 0.181 & 17 & 48.860 & $<0.001$ \\
\hline inter-trocánter & 0.638 & 17 & 89.615 & $<0.001$ \\
\hline
\end{tabular}

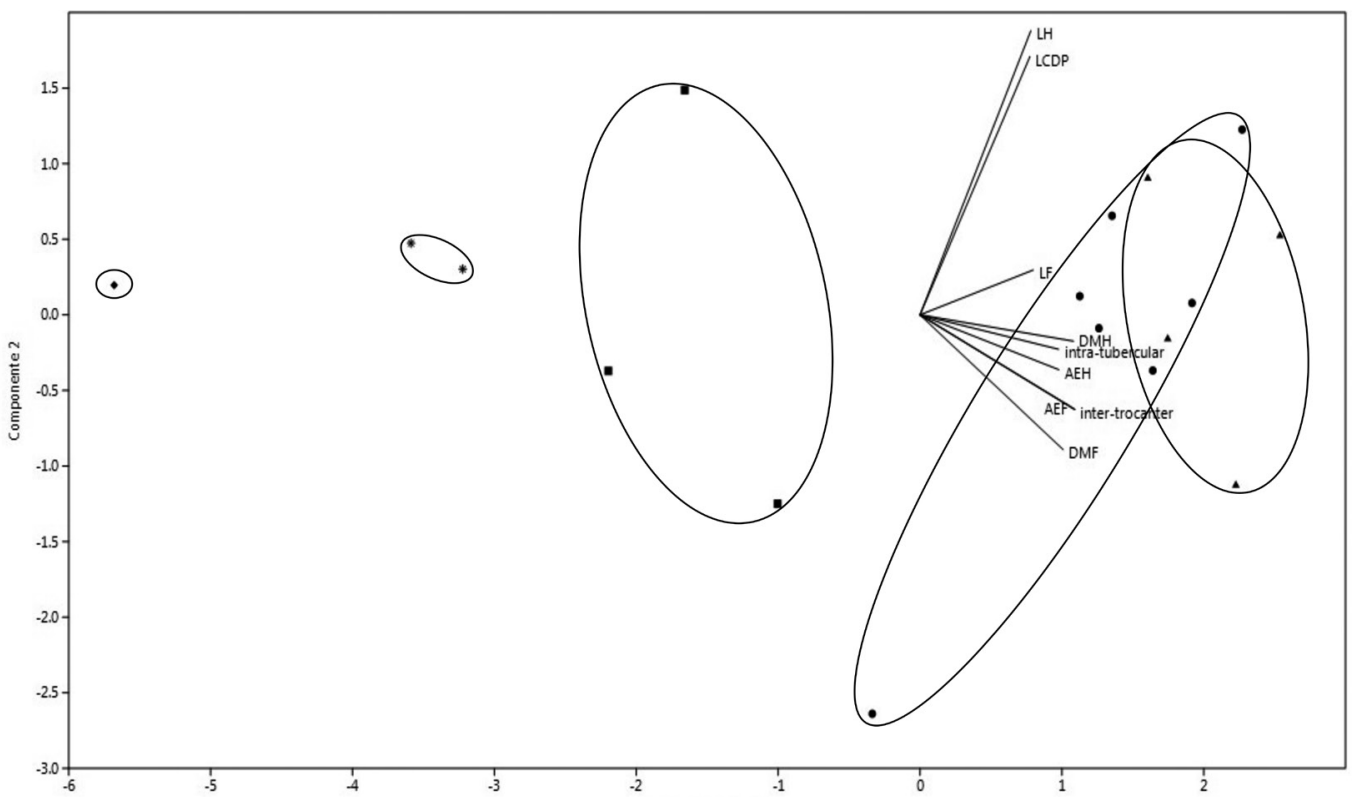

Figura 2 Componentes 1 y 2 (PCA) de las nueve medidas obtenidas en húmero y fémures de Cryptotis de Ecuador. Triángulos = $C$. niausa (fósil), círculos $=C$. niausa (actuales), cuadrados $=C$. montivagus, estrellas $=C$. equatoris y diamante $=C$. osgoodi.

y más anchas sean las estructuras de los extremos de estos dos huesos, mayor es su capacidad como excavador (Woodman y Gaffney, 2014).

En promedio, la forma fósil de C. niausa presentó los tubérculos mayor y menor del húmero (inter-tubercular $=2.81 \mathrm{~mm})$, más grandes respecto a la actual (inter-tubercular $=2.64 \mathrm{~mm}$ ), así también al presentar un mayor índice epicóndilo-femoral (Tabla 3), tiene una mayor fuerza en la flexión de los dedos del pie y el pie. El fósil de C. niausa posee en promedio un fémur más largo $(9.87 \mathrm{~mm})$ que la forma actual $(9.53 \mathrm{~mm})$.

\subsection{HÚMERO}

El húmero de las especies de Cryptotis del Ecuador presenta la forma clásica de los sorícidos con hábitos excavadores (Woodman y Gaffney, 2014). Con húmeros largos, el tubérculo teres desarrollado y en posición medial en el cuerpo del húmero (Figura 3), epicóndilos lateral y medial anchos, procesos tuberculares de la cabeza del húmero expandidos lateralmente y el diámetro del cuerpo del húmero robusto (Bickelmann et al., 2014; Woodman y Gaffney, 2014; He et al., 2015). 
Tabla 3. Medidas obtenidas a los húmeros y fémures de Cryptotis del Ecuador. Mostrando: el promedio, la desviación estándar ( \pm ), valores máximos y mínimos de la medida y entre paréntesis el número de muestras.

\begin{tabular}{|c|c|c|c|c|c|}
\hline Medidas (mm) & C. niausa (fósil) & C. niausa (actual) & C. montivagus & C. equatoris & C. osgoodi \\
\hline LH & $\begin{array}{c}8.75 \pm 0.4 \\
8.23-9.16(4)\end{array}$ & $\begin{array}{c}8.58 \pm 0.4 \\
7.75-9.11(7)\end{array}$ & $\begin{array}{c}8.43 \pm 0.2 \\
8.23-8.67(3)\end{array}$ & $\begin{array}{c}8.14 \pm 0.0 \\
8.10-8.17(2)\end{array}$ & 7.95 \\
\hline LCDP & $\begin{array}{c}4.21 \pm 0.2 \\
4.06-4.5(4)\end{array}$ & $\begin{array}{c}4.26 \pm 0.2 \\
3.77-4.56(7)\end{array}$ & $\begin{array}{c}3.92 \pm 0.4 \\
3.55-4.35(3)\end{array}$ & $\begin{array}{c}3.98 \pm 0.0 \\
3.95-4(2)\end{array}$ & 3.7 \\
\hline AEH & $\begin{array}{c}3.720 .2 \\
3.61-3.95(4)\end{array}$ & $\begin{array}{c}3.87 \pm 0.1 \\
3.81-3.96(7)\end{array}$ & $\begin{array}{c}3.38 \pm 0.3 \\
3.13-3.64(3)\end{array}$ & $\begin{array}{c}3.42 \pm 0.1 \\
3.35-3.49(2)\end{array}$ & 3.01 \\
\hline DMH & $\begin{array}{c}1.06 \pm 0.1 \\
1-1.14(4)\end{array}$ & $\begin{array}{c}1 \pm 0.0 \\
0.96-1.02(7)\end{array}$ & $\begin{array}{c}0.90 \pm 0.1 \\
0.83-0.98(3)\end{array}$ & $\begin{array}{c}0.80 \pm 0.0 \\
0.79-0.81(2)\end{array}$ & 0.78 \\
\hline inter-tubercular & $\begin{array}{c}2.81 \pm 0.2 \\
2.63-3.04(4)\end{array}$ & $\begin{array}{c}2.64 \pm 0.1 \\
2.56-2.75(7)\end{array}$ & $\begin{array}{c}2.44 \pm 0.1 \\
2.32-2.53(3)\end{array}$ & $\begin{array}{c}2.35 \pm 0.0 \\
2.33-2.36(2)\end{array}$ & 2.31 \\
\hline LF & $\begin{array}{c}9.87 \pm 0.4 \\
9.53-10.27(4)\end{array}$ & $\begin{array}{c}9.53 \pm 0.4 \\
8.9-10.15(7)\end{array}$ & $\begin{array}{c}9.43 \pm 0.1 \\
9.35-9.52(3)\end{array}$ & $\begin{array}{c}9.26 \pm 0.0 \\
9.22-9.29(2)\end{array}$ & 8.74 \\
\hline $\mathrm{AEF}$ & $\begin{array}{c}2.69 \pm 0.1 \\
2.53-2.81(4)\end{array}$ & $\begin{array}{c}2.61 \pm 0.0 \\
2.55-2.65(7)\end{array}$ & $\begin{array}{c}2.29 \pm 0.0 \\
2.26-2.32(3)\end{array}$ & $\begin{array}{c}2.13 \pm 0.0 \\
2.10-2.15(2)\end{array}$ & 2.05 \\
\hline DMF & $\begin{array}{c}1.05 \pm 0.1 \\
0.97-1.14(4)\end{array}$ & $\begin{array}{c}1.05 \pm 0.0 \\
1.02-1.08(7)\end{array}$ & $\begin{array}{c}1 \pm 0.1 \\
0.93-1.06(3)\end{array}$ & $\begin{array}{c}0.80 \pm 0.0 \\
0.79-0.80(2)\end{array}$ & 0.7 \\
\hline inter-trocánter & $\begin{array}{c}2.67 \pm 0.1 \\
2.6-2.82(4)\end{array}$ & $\begin{array}{c}2.70 \pm 0.1 \\
2.63-2.77(7)\end{array}$ & $\begin{array}{c}2.41 \pm 0.0 \\
2.40-2.43(3)\end{array}$ & $\begin{array}{c}2.30 \pm 0.1 \\
2.25-2.35(2)\end{array}$ & 2 \\
\hline
\end{tabular}

Uno de los índices que señalan el grado de hábito excavador de las especies de Cryptotis es la relación que existe entre el ancho epicondilar y la longitud del húmero (índice epicóndilo-humeral), en el cual se puede apreciar que mientras mayor sea este índice, mayor es el grado del hábito excavador que tenga Cryptotis, debido principalmente a que los músculos flexores de los dedos de la mano se anclan en el epicóndilo medial y son los que le permiten al animal cavar en el suelo para retirar la tierra al momento de hacer orificios (Woodman y Gaffney, 2014). En este estudio presentamos la dimensión entre el ancho epicondilar, la longitud y el ancho inter-tubercular del húmero (Figura 4). Es importante considerar, el ancho inter-tubercular, pues el tamaño de los tubérculos mayor y menor del húmero junto con el proceso pectoral, nos muestra un mayor desarrollo de las inserciones de los deltoides (Bickelmann et al., 2014), que son los músculos que mueven el brazo, incrementando su fuerza para poder escarbar.

Las medidas cuya significancia diferenciaron los húmeros de la forma fósil y actual de C. niausa en el ANOVA, fueron: el ancho inter-tubercular del húmero $(p=0.038)$ y aunque menos significante el ancho epicondilar $(p=0.041)$.

\subsection{FÉMUR}

El fémur de las especies de Cryptotis de Ecuador es pequeño y alargado. En la Figura 5, podemos observar a uno de los ejemplares de $C$. niausa fósil, el cual presentó un mayor índice epicóndilo-femoral $(\mathrm{IEF}=0.294)$ que los fémures de las poblaciones actuales de la misma especie. Según este índice, $C$. niausa es la especie con una mayor capacidad cavadora entre los Cryptotis de Ecuador, seguida por C. montivagus y C. osgoodi, que es la especie ecuatoriana más pequeña, y que a pesar de tener un fémur corto, posee muy desarrollados los cóndilos femorales (medial y lateral), por esta razón presenta un índice más elevado que $C$. equatoris cuyo IEF es superior (Figura 5).

La proporción entre el ancho inter-trocánter con la longitud del fémur de C. niausa, es mayor que las otras especies estudiadas. Esto indica que en C. niausa los trocánteres son más robustos para la inserción de fuertes músculos que flexionan la cadera y rotan externamente al muslo (Sisson y Grossman, 1982). 


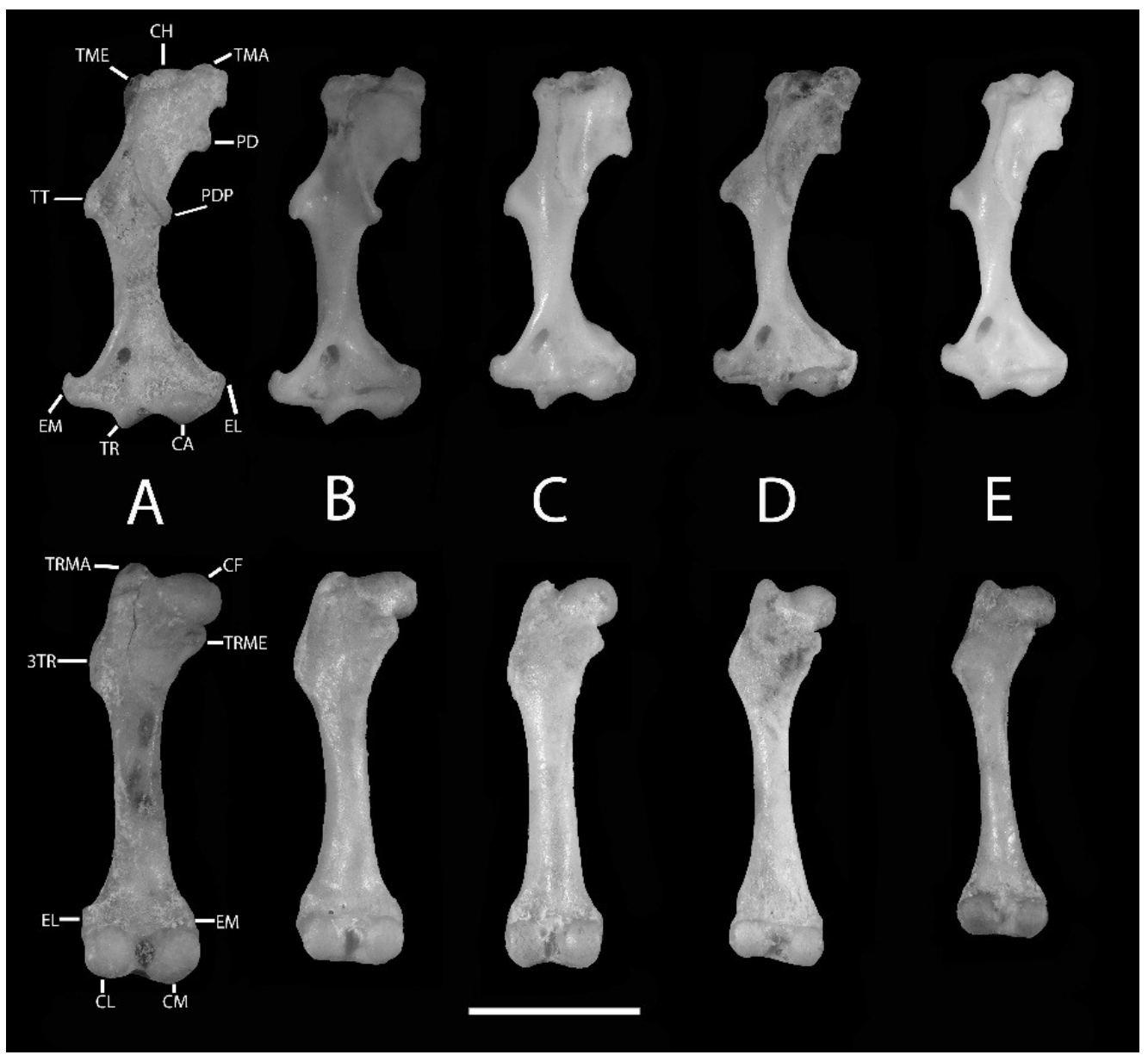

Figura 3 Vista anterior de los húmeros izquierdos (fila superior) $\mathrm{A}=$ C. niausa MEPNV6297 (fósil), $\mathrm{B}=$ C. niausa MEPN9627 holotipo (actual), $\mathrm{C}=\mathrm{C}$. montivagus MEPN10614, $\mathrm{D}=$ C. equatoris MEPN3030 y E=C. osgoodi MEPN6928. Vista posterior de los fémures izquierdos (fila inferior), $\mathrm{A}=\mathrm{C}$. niausa MEPNV6303 (fósil), $\mathrm{B}=\mathrm{C}$. niausa MEPN9627 holotipo (actual), $\mathrm{C}=\mathrm{C}$. montivagus $\mathrm{MEPN} 10616, \mathrm{D}=$ C. equatoris MEPN3030 y E= C. osgoodi MEPN6928. Tubérculo Menor (TME), Cabeza del Húmero (CH), Tubérculo Mayor (TMA), Proceso Deltoide (PD), Proceso Delto Pectoral (PDP), Tubérculo Teres (TT), Epicóndilo Medial (EM), Tróclea (TR), Capitulum (CA), Epicóndilo Lateral (EL), Trocánter Mayor (TRMA), Cabeza del Fémur (CF), Trocánter Menor (TRME), Tercer Trocánter (3TR), Cóndilo Lateral (CL) y Cóndilo Medial (CM). Escala $=4 \mathrm{~mm}$.

\subsection{FRAGMENTO GRANEAL Y MOLARIFORMES O4, M1 Y M2}

El fragmento del maxilar (parte del hueso nasal y el palatino), resume las características propias de C. niausa. En comparación con el resto de especies ecuatorianas, el fragmento del maxilar es grande, similar a C. niausa actual. La parte posterior del palatino del fósil de $C$. niausa es similar a $C$. niausa de la actualidad por poseer el segmento anterior de la fosa mesopterigoidea de forma arqueada (adentrada en el palatino), siendo la única especie del
Ecuador, que presenta esta característica (Figura 6).

El P4 de C. niausa tanto fósil como actual es similar. La forma fósil presenta el protocono más expandido lingualmente que la actual (Figura 6), sin llegar a ser tan expandido como el de C. montivagus (Moreno y Albuja, 2014, Figura 7). Además, de poseer un parastilo reducido (Figura 6), siendo esta variación exhibida también por algunos ejemplares de C. niausa actuales.

El M1 y M2 de Cryptotis niausa fósil al igual que el actual no presentan mayores modificaciones en su 


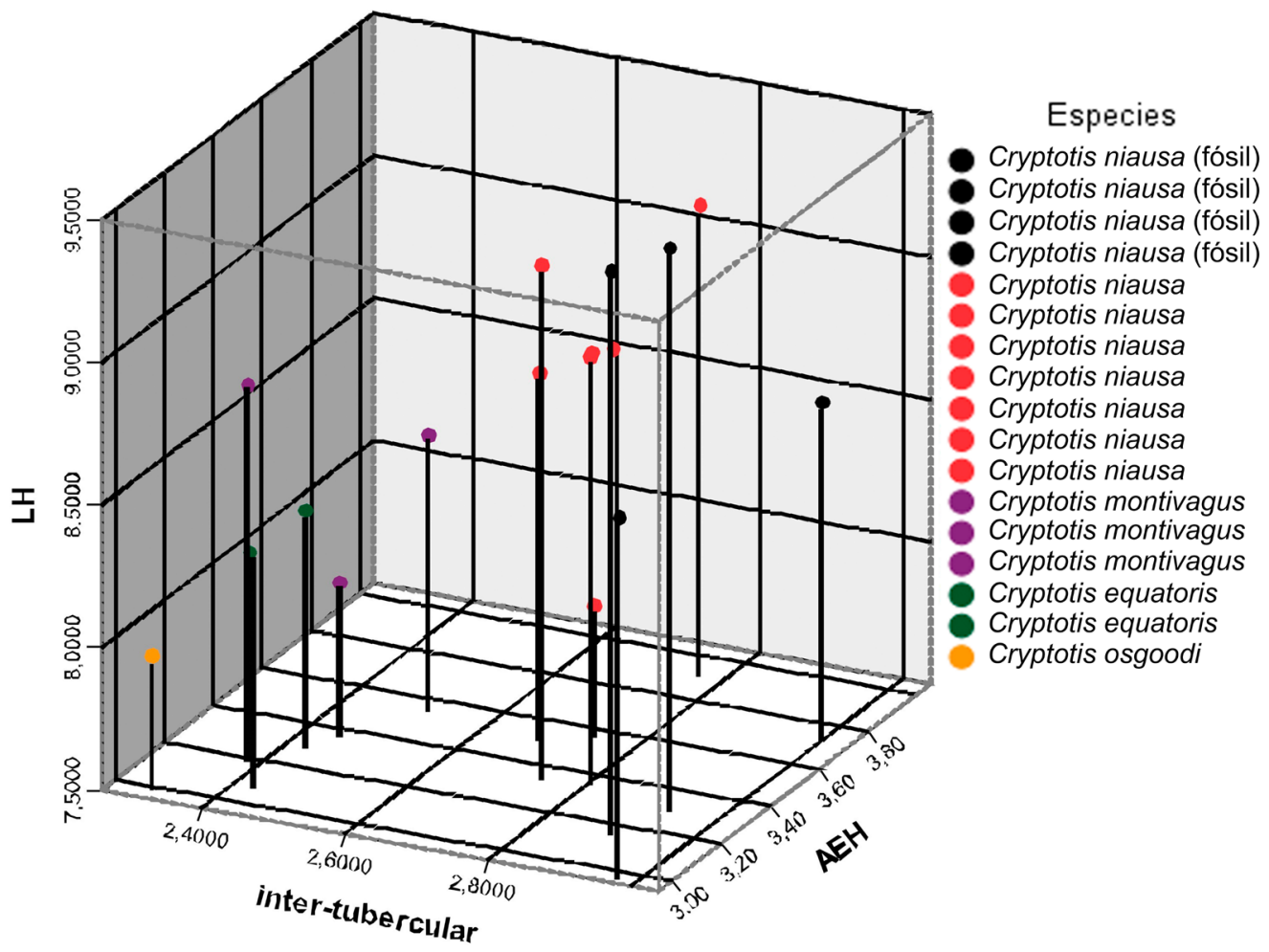

Figura 4 Distribución de las proporciones entre la longitud del húmero (LH), ancho proximal del húmero (inter-tubercular) y ancho epicondilar (AEH).

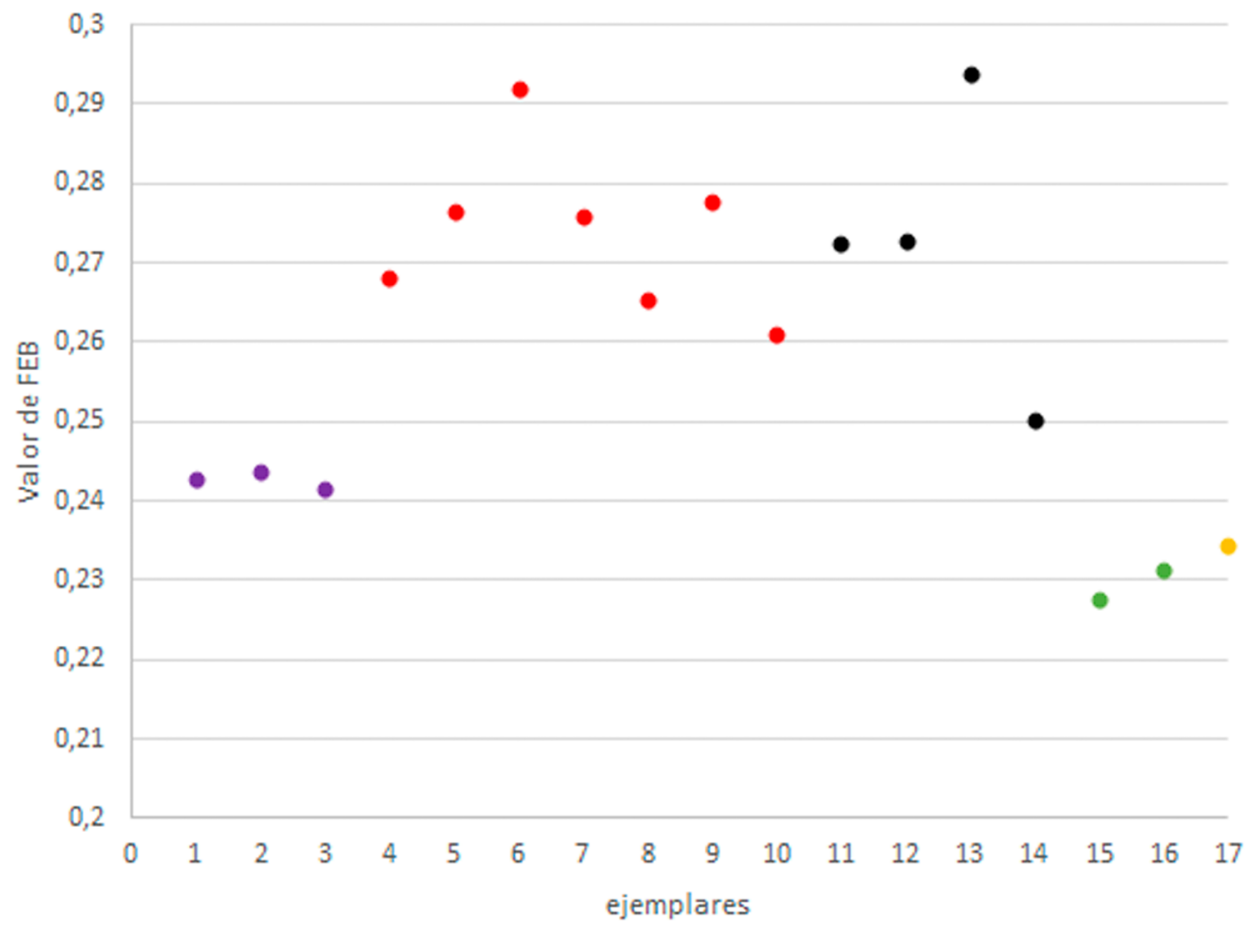

\section{Especies}

Cryptotis niausa (fósil)

Cryptotis niausa (fósil)

Cryptotis niausa (fósil)

Cryptotis niausa (fósil)

Cryptotis niausa

- Cryptotis niausa

- Cryptotis niausa

Cryptotis niausa

Cryptotis niausa

Cryptotis niausa

- Cryptotis niausa

- Cryptotis montivagus

Cryptotis montivagus

- Cryptotis montivagus

- Cryptotis equatoris

Cryptotis equatoris

Cryptotis osgoodi

Figura 5 Índice epicóndilo-femoral (IEF) de las especies de Cryptotis de Ecuador. 


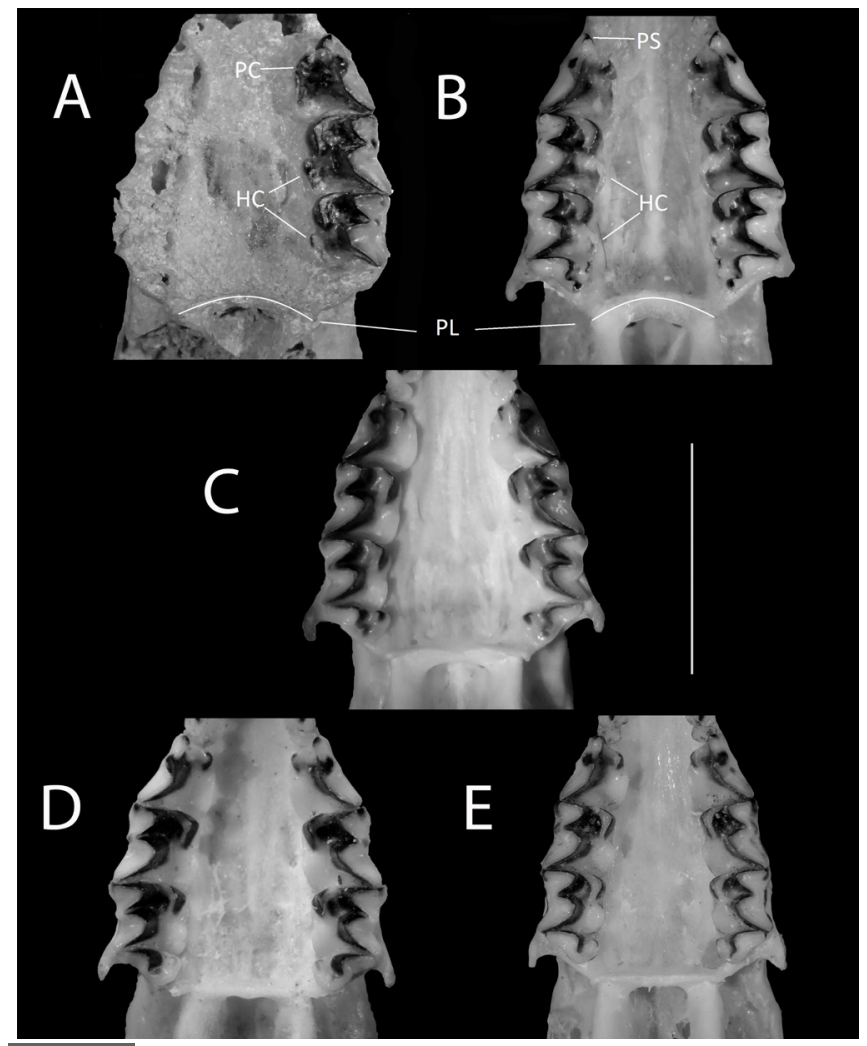

Figura 6 Vista oclusal de la región palatal de Cryptotis del Ecuador con el detalle de sus estructuras diagnósticas. $\mathrm{A}=C$. niausa MEPNV6292 (fósil), B= C. niausa MEPN9627 holotipo (actual), $C=C$. montivagus MEPN12499, $D=C$. equatoris MEPN3021 y E $=$ C. osgoodi MEPN6928. Paracono (PC), Parastilo (PS), Hipocono (HC), Borde posterior palatal (PL). Escala $=5 \mathrm{~mm}$.

estructura. En el M1 el elemento anterior del ectolofo es menor que el posterior (Moreno y Albuja, 2014), con los protoconos e hipoconos alargados lingualmente, especialmente el hipocono. El M2 de C. niausa (fósil y actual) es más grande que las otras especies ecuatorianas, pudiendo alcanzar el mismo tamaño que el M1 (Figura 6), mientras que el M2 en las otras especies es notoriamente menor que el M1.

\section{Discusión}

El registro fósil de C. niausa en la localidad de Quebrada Chalán en la provincia de Chimborazo nos muestra que en el pleistoceno tardío ( 41 ka) esta especie se distribuía más al sur de los páramos donde habita hoy en día. Los hábitats altoandinos de los alrededores de Chalán actualmente están ocupados por: C. osgoodi a $78 \mathrm{~km}$ al norte de Quebrada Chalán (Moreno y Albuja, 2014), mientras que a $66 \mathrm{~km}$ al sur de la misma localidad habita C. montivagus al sur de la provincia de Chimborazo (Lee et al., 2011). Cryptotis niausa hoy se distribuye desde Papallacta en la cordillera oriental de las provincias de Pichincha y Napo hasta Guanderas y la Reserva Ecológica "El Ángel" en la cordillera occidental y oriental de la provincia del Carchi, y tiene preferencia por los hábitats de páramo de pajonal húmedo, sobre los 3600 m (Moreno y Albuja, 2014).

El sector de Quebrada Chalán es un área muy intervenida por las diferentes actividades humanas, cubierta de poca vegetación arbórea y que recibe poca pluviosidad; y en este tipo de hábitat hasta el momento no se han registrado especímenes del género Cryptotis en Ecuador (Moreno y Albuja, 2014).

Por consiguiente el yacimiento pleistocénico de Quebrada Chalán, durante el tercer interglacial debió haber presentado vegetación de gramíneas junto con remanentes boscosos (Mesías, 2012; Sánchez et al., 2013), similar a los páramos actuales, pero con una altura menor a la que se distribuyen actualmente (> 3200 m) en los Andes de Ecuador (MAE, 2013); lo cual sirvió también de refugio para algunas especies de megafauna (Hoffstetter, 1952; Ficcarelli et al.,1992, 1997) donde compartieron hábitat con C. niausa. Esta conclusión es apoyada además por las especies de roedores fósiles que fueron identificados en el mismo yacimiento: Microryzomys altissimus, Akodon mollis, Phyllotis andium, Thomasomys aureus y T. baeops, Sigmodon inopinatus (Cricetidae), que son propias de ambientes paramunos (Patton et al., 2015) y por fitolitos de gramíneas que predominaban en el suelo de esa época (Sánchez et al., 2013).

$\mathrm{El}$ análisis morfológico de C. niausa fósil nos muestra que las poblaciones pleistocénicas poseían una estructura ósea y muscular más robusta que las poblaciones actuales, presentándose en algunos individuos dimensiones mayores de las estructuras de los húmeros y los fémures (Tabla 3). Nuestras 
observaciones concuerdan con Choate (1970), Esteva et al. (2005) y Woodman y Croft (2005) quienes mencionan que las variaciones de tamaño en los registros fósiles de las musarañas del género Cryptotis dependen por una parte de los factores relacionados con los cambios de temperatura y humedad, y por otra, de la disponibilidad de alimentos, competitividad y hábitos de las especies en las distintas etapas del pleistoceno-holoceno.

En el pleistoceno superior de Quebrada Chalán, se sedimentaron cenizas endurecidas y materiales ferruginosos, típicos de suelos con actividad volcánica y en un ambiente con progresiva disminución de la pluviosidad (Sauer, 1955; Sánchez et al., 2013). La forma y dimensiones humerales y femorales de $C$. niausa sugieren que habitó ecosistemas con suelos duros, por lo que tenía que esforzarse más para cavar sus madrigueras y canales.

En el presente tiempo, C. niausa tiene mayores adaptaciones en el húmero y el fémur que estaría asociado al ejercicio de excavación, por sus desarrollados cóndilos humerales y femorales, así como amplios tubérculos, cresta deltopectoral del húmero y trocánteres en el fémur, lo que le permite poder excavar el suelo duro de los páramos herbáceos. Mientras que en C. montivagus, esta capacidad es menor, por cuanto el suelo de los páramos arbustivos y bosques montanos andinos donde habita está cubierto por musgos y hojarasca, es decir, una textura más suave por donde movilizarse.

La fuerza para excavar de $C$. equatoris disminuye en relación con las anteriores especies, y puede estar relacionado a la consistencia del suelo de los bosques nublados y montanos andinos donde habita, el cual está compuesto en su mayoría por hojarasca en descomposición.

Cryptotis osgoodi es la especie con el hábito de movilización más ambulatorio entre las especies ecuatorianas, por tener las dimensiones más pequeñas en las estructuras humerales y femorales. Esta especie habita los bosques montanos de las estribaciones orientales de los Andes y valles interandinos en Ecuador, y es una especie pequeña y delgada (Moreno y Albuja, 2014) que habita ecosistemas con suelo blando o con galerías subterráneas na- turales, por lo que posiblemente no desarrolló una mayor capacidad cavadora.

\section{Agradecimientos}

Expresamos nuestros agradecimientos a Katherine Moreno, de la Facultad de Geología de la EPN, por su importante colaboración en la elaboración del mapa de distribución de C. niausa. A Santiago Ron, Gurador de Anfibios del Museo de Zoología de la Pontificia Universidad Católica del Ecuador (QCAZ). Un agradecimiento especial a Miguel Pinto, Encargado del Instituto de Ciencias Biológicas de la Escuela Politécnica Nacional del Ecuador (MEPN), por la revisión y comentarios a este trabajo. A Alejandro Mesías Moreno por la facilitación del material de los fósiles de micromamíferos del sector de quebrada Chalán. A Mateo Bohórquez por la revisión del resumen en inglés. A Marisol Montellano de la Universidad Nacional Autónoma de México y Marcial Quiroga-Carmona del Centro de Ecología del Instituto Venezolano de Investigaciones Científicas por las revisiones, correcciones y comentarios al manuscrito.

\section{Referencias}

Andrews, P., 1990, Owls, caves and fossils: Chicago-London, The University of Chicago Press, 231 p.

Bickelmann, C., Jiménez, R., Richardson, M.R., Sánchez-Villagra, 2014, Humerus development in moles (Talpidae, Mammalia): Acta Zoologica, 95, 283-289.

Choate, J.R., 1970, Systematics and Zoogeography of Middle American shrews of the Genus Cryptotis: University of Kansas Publications, Museum of Natural History, 19, 195-317.

de Porta, J., 1965, Nota preliminar sobre la fauna de vertebrados hallada en Curití (Departamento de Santander, Colombia): Boletín de Geología, 19, 112-115. 
Esteva, M.J., Arroyo-Cabrales, A.F., Martinez, E., Johnson, Polaco, O.J., 2005, Fossil shrews from San Josecito Cave, Nuevo Leon, Mexico, en Merritt, J., Churchfield, S., Hutterer, R., Sheftel, B. (ed.), Advances in the biology of shrews II: Special Publication of the International Society of Shrew Biologists, 1, 31-48.

Fejfar, O., Blasetti, A., Calderoni, G., Coltorti, M., Ficcarelli, G., Masini, F., Rook, L., Torre, D., 1993, New finds of Cricetids (Mammalia: Rodentia) from the late PleistoceneHolocene of northern Ecuador: Documents des Laboratoires de Lyon, 125, 151-167.

Fejfar, O., Ficcarelli, G., Mezzabotta, C., Moreno, M., Rook, L., Torre, D., 1996, First record of a comyine-peromyscine cricetid (Rodentia: Mammalia) in South America: hypotheses regarding its ancestry in the Paleartic: Acta Zoologica Cracoviensia, 39, 137-145.

Ficcarelli, O., Azzaroli, A., Borselli, V., Coltorti, M., Dramis, E., Fejfar, O., Hirtz, A., Torre, D., 1992, Stratigraphy and paleontology of upper Pleistocene deposits in the Interandean Depression, Northern Ecuador: Journal of South American Earth Sciences, 6, 145-150. Ficcarelli, G., Azzaroli, A., Bertini, A., Coltorti, M., Mazza, P., Mezzabotta, C., MorenoEspinosa, M., Rook, L., Torre, D., 1997, Hypothesis on the cause of extinction of the South American Mastodonts: Journal of South American Earth Sciences 10, 29-38.

Findley, J.S., 1953, Pleistocene Soricidae from San Josecito Cave, Nuevo Leon, Mexico: University of Kansas Publications, Museum of Natural History, 5(36), 633-639.

Hammer, Ø., Harper, D.A., Ryan, P., 2001, PAST: Paleontological Statistics Software Package for Education and Data Analysis: Palaeontologia Electronica, 4(1), 1-9.

Harris, A.H., 1989, The New Mexican late Wisconsineast versus west: National Geographic Research, 5, 205-217.
Harris, A.H., 1997, Geographic and chronologic patterns in Late Pleistocene vertebrate faunas, southern New Mexico, en Lucas, G., Estep, J., Williamson, T., Morgan, G. (ed), New Mexico's Fossil Record 1: New Mexico Museum of Natural History and Science Bulletin, 11, 129-134.

He, K., Woodman, N., Boaglio, S., Roberts, M., Supekar, S., Maldonado, J., 2015, Molecular Phylogeny Supports Repeated Adaptation to Burrowing within Small-Eared Shrews Genus of Cryptotis (Eulipotyphla, Soricidae); PLoS ONE, 10(10), 1-13.

Hoffstetter, R., 1952, Les mammifères Pléistocènes de la République de l'Equateur: Mémoires de la Societe geologique de France, 31, 1-391.

Josse, C., Cuesta, F., Navarro, G., Barrena, V., Cabrera, E., Chacón-Moreno, E., Ferreira, W., Peralvo, M., Saito, J., Tovar, A., 2009, Mapa temático, Mapa de Ecosistemas de los Andes del Norte y Centro. Bolivia, Colombia, Ecuador, Perú y Venezuela, 1:4100000, Lima, Perú, Secretaría General de la Comunidad Andina, Programa Regional ECOBONA, CONDESAN-Proyecto Páramo Andino, Programa BioAndes, EcoCiencia, NatureServe, LTA-UNALM, IAvH, ICAELA, GDCUNALM, RUMBOL SRL.

Lee, T., Boada, C., Scott, A., Burneo, S., Hanson, J., 2011, Small mammals of Sangay National Park, Chimborazo Province and Morona Santiago Province, Ecuador: Occasional Papers of Museum Texas, Tech University, $305,1-14$.

Ministerio del Ambiente del Ecuador (MAE), 2013, Sistema de Clasificación de los Ecosistemas del Ecuador Continental: Quito, Subsecretaría de Patrimonio Natural, Quito, $235 \mathrm{p}$.

Mesías, A., 2012, Registros y distribución de roedores fósiles pertenecientes al Pleistoceno de Ecuador: Quito, Ecuador, Universidad central del Ecuador, Tesis de Licenciatura, $100 \mathrm{p}$. 
Moreno, P.A., Albuja, L., 2014, Una nueva especie de Musaraña del género Cryptotis Pomel 1848 (Mammalia: Soricomorpha: Soricidae) de Ecuador y estatus taxonómico de Cryptotis equatoris Thomas (1912): Papéis Avulsos de Zoologia, 54(28), 403-418.

Patton, J.L., Pardiñas, U.F.J., D’Elia, G. (eds), 2015, Mammals of South America: Volume 2: Rodents: Chicago, USA, University Chicago Press, 1336 p.

Román-Carrión, J.L., 2010, Reanudación de investigaciones paleontológicas en el yacimiento pleistocénico de Punín, Quebrada de Chalán, provincia de Chimborazo, Ecuador, en Artabe, A., Ballent, S., Candela, A., Iglesias, A., Tortello, F. (eds), X Congreso Argentino de Paleontología y Bioestratigrafía y VII Congreso Latinoamericano de Paleontología: La Plata, Argentina, 125-126.

Román-Carrión, J., Lara, P., 2011, La Fauna Fósil de Quebrada Chalán: Ecuador Fósil, fascículo N.-1, Escuela Politécnica Nacional, Quito-Ecuador, 38 p.

Sánchez, M.V., Genise, J., Bellosi, E., RománCarrión, J.L., Cantil, L., 2013, Dung beetle brood balls from Pleistocene highland palaeosols of Andean Ecuador: A reassessment of Sauer's Coprinisphaera and their palaeoenvironments: Palaeogeography Palaeoclimatology Palaeoecology, 386, 257-274.

Sauer, W., 1950, Contribuciones para el Conocimiento del Cuaternario en el Ecuador: Anales de la Universidad Central del Ecuador, LXXVII, 328, 326-364.

Sauer, W., 1955, Coprinsphaera ecuadoriensis un fósil singular del Pleistoceno: Boletín del Instituto de Ciencias Naturales de la Universidad Central de Quito, 1(2), 123-129.

Sisson, S., Grossman, J.D., 1982, Anatomía de los animales domésticos: Barcelona, Salvat, 952 p.

SPSS, 2009, PASW statistics 18.0. Version 18.0. Environmental System Research Institute: Armonk, New York.
Woodman, N., Croft, D., 2005, Fossil shrews from Honduras and their significance for late glacial evolution in body size (Mammalia: Soricidae: Cryptotis): Fieldiana: Geology, New Series, 1534, 1-30.

Woodman, N., Gaffney, S., 2014, Can they dig it? Functional morphology and degrees of semifossoriality among some American shrews (Mammalia, Soricidae): Journal of Morphology, 275, 745-759.

Woodman, N., Stabile, F., 2015, Variation in the Myosoricinae hand skeleton and its implications for locomotory behavior (Eulipotyphla: Soricidae): Journal of Mammalogy, 96(1), $159-171$

\section{Apéndice}

Especímenes examinados: en total se examinaron 13 especímenes con húmeros y fémures completos de Cryptotis actuales de Ecuador y 10 huesos fósiles, todos del Instituto de Ciencias Biológicas de la Escuela Politécnica Nacional del Ecuador (MEPN)

Cryptotis equatoris: Pichincha: parroquia Calacalí, "Loma La Liberia", 00'30.66"N/78³2'4.87'O, 2500 msnm, (MEPN3021, MEPN3030).

Cryptotis montivagus: Azuay: Cercanías de Cuenca, 255'5.44"S/ $79^{\circ}$ 4'53.68”O, 2650 msnm (MEPN10614). Loja: P.N. Podocarpus, lag. "El Compadre", 04²10’S 7907’O, 2900-3200 msnm, (MEPN10616); Cantón Saraguro, Sabadel, "Cerro de Arcos", 0337'4.57'S / 79²9'18.00”O, 2403 msnm (MEPN12499 ぶ).

Cryptotis niausa (actual): Carchi: Cantón Espejo, La Libertad, Hostería Polylepis, $\quad 0^{\circ} 43^{\prime} 11.85$ ”N/7758'11.93”O, $3640 \mathrm{msnm}$, (MEPN10695, MEPN10876,JBM25D1). Páramos del Ángel, 043'15.16”N/7756'34.8”O, 3600 msnm, (MEPN9790). Napo: Papallacta "La Virgen”, 0²0'49.2”N/78¹2'0”O, 3700 msnm, (MEPN9627 [holotipo], MEPN10618, MEPN9628ㅇ,). Cryptotis niausa (fósil): Chimborazo: Cantón Riobamba, Parroquia Licto, Quebrada Chalán, 01046'32.3"S/78o37'41.1'W, 2800 msnm, (MEPNV6292, MEPV6295 - MEPMV6304)

Cryptotis osgoodi: Pichincha: Alrededores de Quito, 0¹3'18.76”S/78²8'55.38”O, 2816 msnm, (MEPN6928). 